\title{
Josephson vortex interaction mediated by cavity modes: Tunable coupling for superconducting qubits
}

\author{
M. V. Fistul and A. V. Ustinov \\ Physikalisches Institut III, Universität Erlangen-Nürnberg, D-91058 Erlangen, Germany
}

(Dated: September 20, 2018)

\begin{abstract}
A quantum-mechanical model for the interaction of Josephson vortices (fluxons) embedded in superconducting transmission line is presented. The vortices interact through emission and absorption of linear waves (electromagnetic cavity modes). We show that in a classical regime this peculiar type of interaction is determined by the product of instantaneous velocities of fluxons. In a quantum regime, this property provides tunable coupling between vortices which can be used for entanglement of vortex qubits. The physical mechanism of the vortex interaction is similar to that proposed for qubits based on trapped ions. Different types of transmission lines mediating the vortex interaction are proposed.

PACS numbers: 03.67.Lx, 03.75.Lm, 74.50.+r
\end{abstract}

It is well established that Josephson vortices (magnetic fluxons) can be trapped in spatially extended Josephson structures [1, 2]. Moreover, in the presence of an externally applied magnetic field and dc bias these peculiar nonlinear objects propagate along a system or are trapped around a particular point $[2,[3,4,[5]$. In a classical regime the vortex motion is mapped to the classical mechanics of a macroscopic particle. As we turn to a quantum regime realized at low temperatures and for a small energy of the vortex in an external potential, the vortex is predicted to behave quantum-mechanically, e. g. show tunneling effect and discrete energy levels $[\underline{6}, 7]$. Quantum tunneling of vortices has been observed in discrete arrays of small Josephson junctions [8], and recently quantum tunneling of a single vortex was observed in long annular Josephson junctions 9]. A quantum state of a macroscopic vortex in an artificially created potential can be used in order to implement a particular type of superconducting qubits named vortex qubits [10, 11].

Up to now, two types of vortex qubit prototypes have been investigated. The first prototype is the heartshaped Josephson junction shown in Fig. 17, for which rather simple state preparation and readout procedures have been already verified experimentally 11]. In these qubits the external magnetic field is uniform and the spatially-dependent potential profile for a vortex is tailored by shaping the long junction into a heart form. The second vortex qubit idea is based on creating a desired potential for the vortex by using local magnetic fields 12] generated by control currents $I_{1}$ and $I_{2}$, see Fig. 10. Both cases can be described by a local double-well potential $U\left(y_{i}\right)$ for the vortex, see Fig. 11. Here, $y_{i}$ is the coordinate of the center of the vortex.

The qubit shown in Fig. 10 has the advantage that its state preparation and readout can be done by using Rapid Single Flux Quantum (RSFQ) circuits. RSFQ logic is the natural choice for an interface between vortex qubits and room-temperature electronics. The fieldinjector based vortex qubits with RSFQ preparation and read-out interfaces have been also recently tested in the classical regime [13]. The entanglement that persists (a)

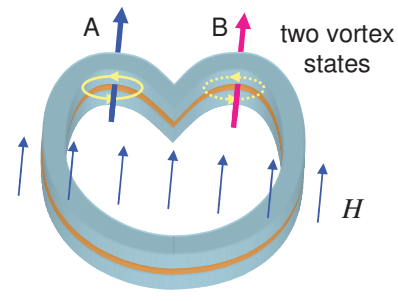

(b)

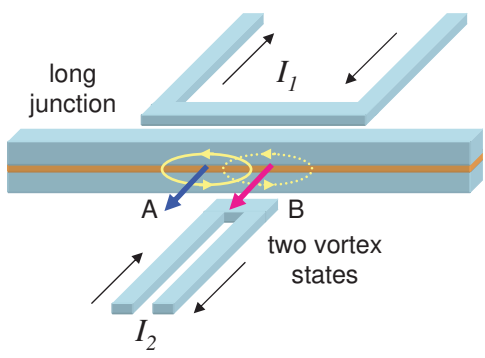

(c)

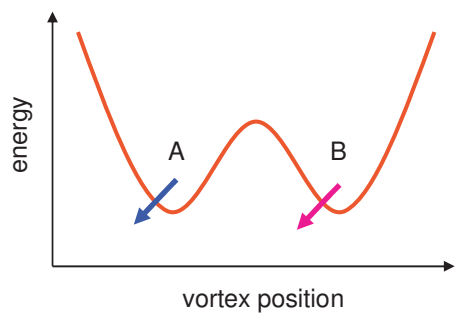

FIG. 1: Possible realizations of vortex qubits. a) Heartshaped long Josephson junction placed in the magnetic field $H$ [1]. b) Long Josephson junction with local magnetic field injectors. c) Double-well potential for a vortex in the above two systems. Two vortex states are indicated as A and B.

in the quantum regime in a multi-qubit circuit should be possible to read out by RSFQ circuitry. The vortex qubits are naturally scalable to larger quantum circuits.

In a system containing a number of vortices they interact with each other. The simplest type of the interaction, namely direct static magnetic interaction, occurs 
when the vortices are located in the same long Josephson junction, see Fig. 2k. In this case the interaction is due to the presence of a mutual inductance between the two vortex locations and is determined by overlapping of the "tails" of vortex magnetic fields. Such an interaction decays exponentially [14] with the distance between the vortices on the characteristic scale of the Josephson penetration depth $\lambda_{J}$ and depends on their coordinates. In the quantum regime the coupling between vortices leads to a quantum entanglement, which is the necessary condition in order to realize a quantum computation.

The static interaction exponentially depends on the distance between vortices so that only coupling between the nearest neighbors has to be taken into account. In coordinate representation the static interaction term between two vortices can be written as $U_{0} \exp \left(\left|y_{i}-y_{j}\right| / \lambda_{J}\right)$, where $y_{i}, y_{j}$ are the coordinates of the interacting vortices, and $U_{0}$ is the characteristic interaction energy. If we switch to the representation of wave functions corresponding to the two energy levels of single vortices, the interaction term in the Hamiltonian can be written as $\hat{H}_{\text {int }}=\sum U_{0} \exp \left(\left|a_{i}-a_{j}\right|\right) \hat{\sigma}_{z}^{i} \hat{\sigma}_{z}^{j}$, where $a_{i}, a_{j}$ are the average coordinates of corresponding vortices normalized to $\lambda_{J}$. Note here, that with this type of interaction the chain of vortices is mapped to a 1-D Ising model of interacting spins $1 / 2$ or to a system of nuclear spins [15].

A severe drawback of the static type of interaction is that it can not be turned off and addressing of a chosen qubit (pinned vortex) will immediately affect all other qubits. Thus, in this realization it is easy to obtain entangled states but it remains a hard problem to address a single vortex, e.g. switch from the ground state to the excited state $(|0>\rightarrow| 1>$ transition) of a particular vortex without affecting others.

In order to avoid the above problem, we propose for coupling of vortex qubits placing them in a superconducting transmission line (Fig. 2b and 25). In this case the vortices display a peculiar indirect dynamic interaction through virtual excitation (absorption) of linear electromagnetic waves (EW) propagating in a transmission line. This interaction can be realized at least in two different configurations. One is a lateral configuration shown in Fig. 2] , where two or more long Josephson junctions are incorporated in a common system with a superconducting ground plane. In such a system the superconducting electrodes and the ground plane provide a low dissipative transmission line for propagation of EW. Thus, a moving (oscillating) vortex excites the EW (emission of photons) which propagate through the transmission line and interact (by absorption of photons) with other vortices. Note, that this principle allows to realize a quantum-mechanical interaction similar to the one proposed for chains of trapped ions [16, 17]. In the latter system single-qubit operations address ion energy levels but the interaction is just due to the excitation of phonons in a chain of trapped ions. Similar realization of coupling via lumped $L-C$ circuit has been proposed earlier [18, 19] for superconducting qubits. (a)

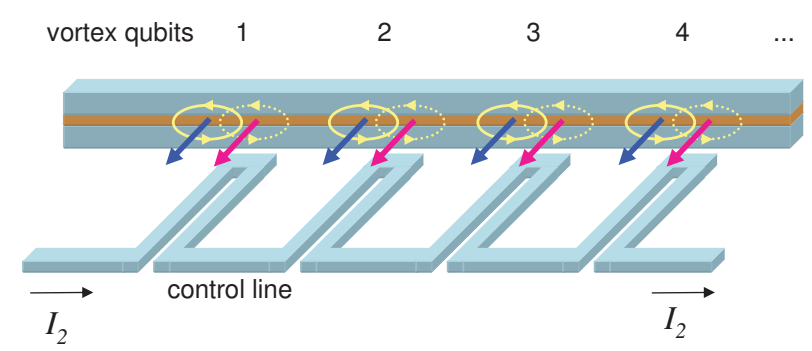

(b)

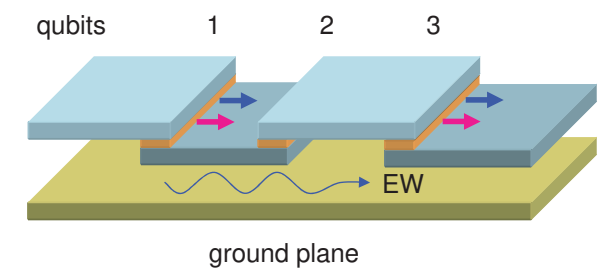

(c)

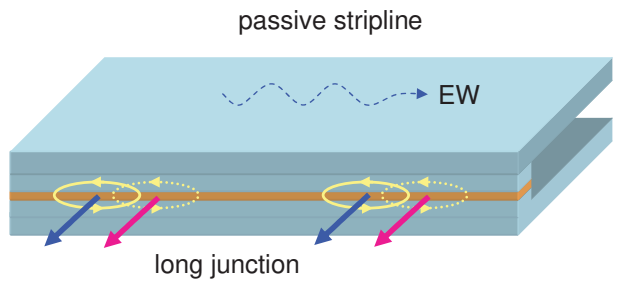

FIG. 2: Sketch of long Josephson junction circuits allowing for different types of vortex interaction. a) Static coupling between vortex qubits can be realized by placing them side by side in a long junction. The distance $d$ between qubits has to be larger (but not much larger) than $\lambda_{J}$. b) The dynamic coupling between vortex qubits located in different junctions. The junctions are placed above a superconducting ground plane. c) The dynamic coupling between vortex qubits separated by large distance in a single long junction. Note a passive stripline behind the junction. It provides the coupling between qubits via electromagnetic waves (EW) propagating in the stripline.

Anther possible realization of the dynamic coupling is a configuration with far-separated vortices located in the same Josephson junction. These vortex qubits indirectly interact with each other through the EW excitations in a passive transmission line inductively coupled to the junction, see Fig. 2r. Similarly to a lateral configuration, the EW propagating in transmission line provides a fast and low dissipative source of interaction.

In the following, we quantitatively analyze the lateral configuration of vortex qubits located in different long junctions placed above a superconducting ground plane (Fig. 2b). A classical dynamics of such a system is determined by time-dependent Josephson phases $\varphi\left(y-y_{i}, t\right)$, where $y$ is the coordinate along Josephson junctions and $y_{i}$ is the coordinate of the center of the vortex located in the $i$-th Josephson junction. The EW propagation along the transverse coordinate $x$ is described by a set of equa- 
tions:

$$
\begin{gathered}
\frac{\partial V(x, t)}{\partial x}=L \frac{\partial I(x, t)}{\partial t}+R_{s} I(x, t)+ \\
+\sum_{i} \int \frac{d y}{a} \frac{\hbar}{2 e} \frac{\partial \varphi\left(y-y_{i}, t\right)}{\partial t} \delta\left(x-x_{i}\right) \\
\frac{\partial I(x, t)}{\partial x}=C \frac{\partial V(x, t)}{\partial t},
\end{gathered}
$$

where $x_{i}$ are the coordinates of Josephson junctions, $a$ is the width of the Josephson junction in $x$ - direction, $V(x, t)$ is the voltage between the superconducting electrodes and the ground plane, $I(x, t)$ is the current flowing in $x$ direction along the superconducting electrodes. The parameters $L$ (the inductance of superconducting electrodes per unit length) and $C$ (the capacitance between superconducting electrodes and the ground plane per unit length) determine the properties of a transmission line created between the superconducting electrodes and the ground plane. The parameter $R_{s}$ describes the dissipation due to the surface losses (in general, they are frequency dependent) in the transmission line. For not very high frequencies these losses in the superconducting line are very low. Notice here, that without the term depending on the Josephson phases this set of equation describes the propagation of a weakly decaying linear EW with the spectrum $\omega(k)=c k$, where $c=1 / \sqrt{L C}[20]$. In the presence of vortices we obtain the wave equation where the vortices are the local sources of perturbation:

$$
\begin{gathered}
\frac{\partial^{2} I(x, t)}{\partial x^{2}}-C L \frac{\partial^{2} I(x, t)}{\partial t^{2}}-R C \frac{\partial I(x, t)}{\partial t}= \\
=-C \frac{\pi \hbar}{a e} \sum_{i} \ddot{y}_{i} \delta\left(x-x_{i}\right) .
\end{gathered}
$$

Thus, we obtain a time Fourier transformation of the ac current distribution in the transmission line $I_{\omega}(x)$ in the form:

$$
I_{\omega}(x)=C \frac{\pi \hbar}{a e} \sum_{i} \omega^{2} G_{\omega}\left(x, x_{i}\right) \int d t e^{i \omega t} y_{i}(t)
$$

where $G_{\omega}\left(x, x_{i}\right) \equiv G_{\omega}\left(x-x_{i}\right)$ is the Green function of the linear equation:

$\frac{d^{2} G_{\omega}\left(x, x_{1}\right)}{d x^{2}}+C L \omega^{2} G_{\omega}\left(x, x_{1}\right)-i \omega R C G_{\omega}\left(x, x_{1}\right)=\delta\left(x-x_{1}\right)$.

The equation of motion for a vortex center of mass in the presence of a local double-well potential $U\left(y_{i}\right)$ illustrated in Fig. 10 is written as

$$
\ddot{y}_{i}+\frac{\pi \lambda_{J} \omega_{p}^{2}}{4}\left[\frac{2 e \lambda_{J}}{\hbar I_{c 0}} \frac{\partial U\left(y_{i}\right)}{\partial y_{i}}-\frac{I\left(x_{i}, t\right)}{I_{c 0}}\right]=0,
$$

where $\omega_{p}$ and $I_{c 0}$ are the plasma frequency and the critical current of a Josephson junction (in the absence of vortices), respectively [3, 4]. Thus, the interaction energy between vortices can be found as:

$$
U_{i n t}=-\sum_{i} \frac{\pi \hbar}{e} \frac{I\left(x_{i}, t\right) y_{i}(t)}{\lambda_{J}} .
$$

By making use of (3) and (4) we obtain

$$
\begin{gathered}
U_{i n t}=\frac{C}{a \lambda_{J}}\left(\frac{\pi \hbar}{e}\right)^{2} \\
\times \sum_{i \neq j} \int d t_{1} \dot{y}_{i}(t) \dot{y}_{j}\left(t_{1}\right) \int d \omega e^{\mathrm{i} \omega\left(t-t_{1}\right)} G_{\omega}\left(x_{i}-x_{j}\right) .
\end{gathered}
$$

This expression can be symmetrized over the indexes $i$ and $j$ as

$$
\begin{gathered}
U_{i n t}=\frac{2 \tau C}{a \lambda_{J}}\left(\frac{\pi \hbar}{e}\right)^{2} \\
\times \frac{1}{2} \sum_{i \neq j} \int d t_{1} \dot{y}_{i}(t) \dot{y}_{j}\left(t_{1}\right) K\left(t-t_{1},\left|x_{i}-x_{j}\right|\right),
\end{gathered}
$$

where the kernel of interaction

$$
K(t, x)=-\mathrm{i} \int d \omega \omega e^{-\mathrm{i} \omega t} G_{\omega}(|x|)
$$

depends on the parameters $L$ and $C$ in our model. In the limit of a small propagation time $\left(\tau=\left|x_{i}-x_{j}\right| \sqrt{L C} \ll\right.$ $T_{\text {osc }}$, where $T_{\text {osc }}$ period of slow vortex oscillations between two quantum states) we neglect a delay in the wave propagation, and obtain the classical interacting potential between vortices located in the Josephson junctions $i$ and $j$ :

$$
U_{i j}=A_{i j} \dot{y}_{i}(t) \dot{y}_{j}(t),
$$

where the constant of interaction $A_{i j}$ is determined by the properties of the transmission line given by (9). For a simplest case of a transmission line described by (2) we obtain

$$
A_{i j}=\frac{\left|x_{i}-x_{j}\right| C}{a \lambda_{J}}\left(\frac{\pi \hbar}{e}\right)^{2} \exp \left(-\frac{R}{2} \sqrt{\frac{C}{L}}\left|x_{i}-x_{j}\right|\right) .
$$

Thus, in the case of an indirect dynamic coupling the vortex interaction is determined by a product of vortex velocities.

In the quantum regime this dynamic interaction is determined by operators of vortex momentum $\left(\hat{p}_{i}\right.$ and $\left.\hat{p}_{j}\right)$ :

$$
\hat{H}_{i n t}=\frac{A_{i j}}{m_{f}^{2}} \hat{p}_{i} \hat{p}_{j}
$$


where $m_{f}$ is the effective mass of a vortex [10]. The total Hamiltonian $H$ of indirectly interacting vortices can be written as

$$
\hat{H}=\sum_{i} \frac{\hat{p}_{i}^{2}}{2 m_{f}}+U\left(y_{i}\right)+\frac{1}{2} \sum_{i \neq j} \frac{A_{i j}}{m_{f}^{2}} \hat{p}_{i} \hat{p}_{j} .
$$

If a single vortex potential $U\left(y_{i}\right)$ has a double-well form, the quantum-mechanical description of a single vortex can be reduced to lowest energy levels in each well. These levels are characterized by the energy difference $\sim B_{z}$ and the tunneling amplitude $\sim B_{x}$ between states [10]. The parameters $B_{z}$ and $B_{x}$ are determined by the properties of a single vortex potential $U\left(y_{i}\right)$. Because the average value of the momentum operator $\left\langle\hat{p}_{i}\right\rangle$ is zero in these states, we obtain that the interaction potential is determined by tunneling amplitudes $B_{x}(i)$. By making use of the spin representation of the Hamiltonian we write

$\hat{H}=\sum_{i}\left[B_{z}(i) \hat{\sigma}_{z}^{i}+B_{x}(i) \hat{\sigma}_{x}^{i}\right]+\frac{1}{2} \sum_{i \neq j} B_{x}(i) B_{x}(j) \tilde{A}_{i j} \hat{\sigma}_{x}^{i} \hat{\sigma}_{x}^{j}$.
A great advantage of this dynamic type of interaction in respect to a direct static one is that the former one is absent when both vortices are in the ground state. With this type of Hamiltonian it is possible to address a single vortex or a bunch of vortices, if that is required [19]. Indeed, by turning off the tunneling amplitude $B_{x}$ of a particular vortex we exclude it from the computation process, but other vortices can still be manipulated.

In conclusion, we proposed here and quantitatively analyzed an interaction of vortices through an exchange of EW propagating in a superconducting transmission line. In a classical regime, this interaction depends on the velocities of vortices. As we turn to a quantum regime, this type of interacting allows for a tunable coupling between vortices that is a crucial condition for implementation of such vortices as superconducting qubits. The addressing of these qubits can be provided by a particular combination of dc pulses allowing to change the parameters $B_{z}$ and $B_{x}$.

We thank A. Kemp for useful remarks on the manuscript and acknowledge discussions with V. K. Kaplunenko, J. Lisenfeld, and A. Wallraff.
[1] A. Barone, and G. Paternó, Physics and Application of the Josephson effect, (Wiley, New York, 1992).

[2] A. V. Ustinov, Physica D, 123, 315 (1998).

[3] N. Gronbech-Jensen, P. S. Lomdahl, and M. R. Samuelsen, Phys. Rev. B 43, 12799 (1991).

[4] A. V. Ustinov, B. A. Malomed, and N. Thyssen, Phys. Lett. A 233, 239 (1997).

[5] M. V. Fistul and A. V. Ustinov, Phys. Rev. B 63, 024508 (2001).

[6] T. Kato and M. Imada, J. Phys. Soc. Jpn. 65, 2963 (1996).

[7] A. Shnirman, E. Ben-Jacob, and B. Malomed, Phys. Rev. B 56, 14677 (1997).

[8] H. S. J. Van der Zant, Phys. Rev. Lett. 66, 2531 (1991).

[9] A. Wallraff, J. Lisenfeld, A. Lukashenko, Y. Koval, M. V. Fistul, and A. V. Ustinov, unpublished (2002).

[10] A. Wallraff, Y. Koval, M. Levitchev, M. V. Fistul, and A. V. Ustinov, J. Low Temp. Phys. 118, 543 (2000)

[11] A. Kemp, A. Wallraff and A. V. Ustinov, Phys. Stat. Sol.(b) 233, 472 (2002).

[12] A. V. Ustinov, Appl. Phys. Lett. 80, 3153 (2002).
[13] V. K. Kaplunenko and A. V. Ustinov, unpublished (2002).

[14] The interacting vortices can be also located in different long Josephson junctions. In this case the direct static magnetic interaction is characterized by the power law but the length of the interaction is much smaller (of the order of the London penetration depth $\lambda_{L}$, as the thickness of the electrodes $d \simeq \lambda_{L}$ ).

[15] S. Lloyd, Scientific American, 273, 140 (1995).

[16] G. P. Berman, G. D. Doolen, R. Mainieri, and V. I. Tsifrinovich, Introduction to Quantum Computers, World Scientific, Singapore (1998).

[17] J. I. Cirac, P. Zoller, Phys. Rev. Lett., 74, 4091 (1995).

[18] Y. Makhlin, G. Schön, and A. Schnirman, Nature, 368, 305 (1999).

[19] Y. Makhlin, G. Schön, and A. Schnirman, Rev. Mod. Phys., 73, 357 (2001).

[20] R. P. Feynman, R. B. Leighton, and M. Sands, The Feynman lectures on physics, Addison-Wesley Reading, Mass. (1989). 